\title{
Treatment of Cryptosporidium: What We Know, Gaps, and the Way Forward
}

\author{
Hayley Sparks ${ }^{1}$ - Gayatri Nair ${ }^{1}$ - Alejandro Castellanos-Gonzalez ${ }^{1}$. \\ A. Clinton White Jr. ${ }^{1}$
}

Published online: 1 August 2015

(C) Springer International Publishing AG 2015

\begin{abstract}
Cryptosporidiosis is increasingly recognized as an important global health concern. While initially reported in immunocompromised patients such as AIDS patients, cryptosporidiosis has now been documented as a major cause of childhood diarrhea and an important factor in childhood malnutrition. Currently, nitazoxanide is the only proven anti-parasitic treatment for Cryptosporidium infections. However, it is not effective in severely immunocompromised patients and there is limited data in infants. Immune reconstitution or decreased immunosuppression is critical to therapy in AIDS and transplant patients. This limitation of treatment options presents a major public health challenge given the important burden of disease. Repurposing of drugs developed for other indications and development of inhibitors for novel targets offer hope for improved therapies, but none have advanced to clinical studies.
\end{abstract}

This article is part of the Topical Collection on Protozoa

A. Clinton White, Jr.

acwhite@utmb.edu

Hayley Sparks

hnsparks@utmb.edu

Gayatri Nair

gvnair@utmb.edu

Alejandro Castellanos-Gonzalez

alcastel@utmb.edu

1 Infectious Disease Division, Department of Internal Medicine, University of Texas Medical Branch Galveston, 301 University Boulevard, Route 0435, Galveston, TX 77555-0435, USA
Keywords Cryptosporidium · Cryptosporidium parvum . Cryptosporidium hominis Cryptosporidiosis $\cdot$ Nitazoxanide Paromomycin

\section{Introduction}

Cryptosporidium species are increasingly recognized as important enteric pathogens $[1,2 \bullet \bullet, 3]$. Cryptosporidiosis was initially recognized as a cause of diarrhea in compromised hosts. Shortly thereafter, zoonotic and waterborne transmission of the parasite was identified. Cryptosporidium is now considered one of the major causes of childhood diarrhea. In addition, Cryptosporidium has been documented as a key component of the vicious cycle of infection and malnutrition that are major contributors to childhood morbidity and mortality worldwide. The majority of human Cryptosporidium infections are attributed to two species: Cryptosporidium hominis and Cryptosporidium parvum [1, 2••]. However, at least 13 other species may infect humans [3-5]. Clinically, cryptosporidiosis causes watery diarrhea in healthy patients. In contrast to other causes, diarrhea caused by cryptosporidiosis tends to be more prolonged and can be chronic in compromised hosts, such as children with malnutrition (Table 1).

Cryptosporidium parasites develop within the microvillus layer of intestinal epithelial cells, mainly found in the small intestines in immunocompetent hosts, but may be found throughout the GI tract and even the respiratory tract. Persistent infection is associated with villus atrophy, crypt hyperplasia, and variable increases in leucocytes in the lamina propria. The symptoms of watery diarrhea and malabsorption are thought to be related to sodium malabsorption, electrogenic chloride secretion, and increased intestinal permeability, and severity of disease correlates with altered intestinal permeability $[6,7]$. These effects are likely mediated by the host 
Table 1 Current and prospective therapeutic options for Cryptosporidium infection

\begin{tabular}{|c|c|c|c|}
\hline & Therapeutics & Research level & Comments \\
\hline \multirow[t]{6}{*}{$\begin{array}{l}\text { Current } \\
\text { therapeutics }\end{array}$} & Nitazoxanide & $\begin{array}{l}\text { FDA approved for treatment } \\
\text { of patients } 1 \text { year and older }\end{array}$ & $\begin{array}{l}\text { Not useful in patients } \\
\text { with AIDS }\end{array}$ \\
\hline & $\begin{array}{l}\text { Nitazoxanide/ } \\
\text { azithromycin/ } \\
\text { paromomycin mixtures }\end{array}$ & $\begin{array}{l}\text { Anecdotal clinical } \\
\text { evidence of response } \\
\text { in transplant patients }\end{array}$ & \\
\hline & CD40 agonist antibody & One clinical study published & $\begin{array}{l}\text { Patients relapsed after } \\
\text { cessation of treatment }\end{array}$ \\
\hline & Paromomycin & Multiple clinical studies & $\begin{array}{l}\text { Clinical results have been } \\
\text { mixed or moderate at bes }\end{array}$ \\
\hline & Azithromycin & One published clinical study & $\begin{array}{l}\text { Decreases parasite load with } \\
\text { clearance in some patient }\end{array}$ \\
\hline & $\begin{array}{l}\text { Rifamycins (rifabutin, } \\
\text { rifaximin) }\end{array}$ & $\begin{array}{l}\text { Several clinical studies which } \\
\text { included AIDS patients }\end{array}$ & $\begin{array}{l}\text { Prevented infection } \\
\text { in AIDS patients }\end{array}$ \\
\hline \multirow[t]{6}{*}{$\begin{array}{l}\text { Prospective } \\
\text { therapeutics }\end{array}$} & CDPK inhibitors & $\begin{array}{l}\text { Several published in vitro } \\
\text { and in vivo studies }\end{array}$ & \\
\hline & $\begin{array}{l}N \text {-Methyl-piperazine- } \\
\text { Phe-homoPhe- } \\
\text { vinylsulfonephenyl } \\
\text { (K11777) }\end{array}$ & Published in vivo studies & $\begin{array}{l}\text { Inhibits clan CA cysteine } \\
\text { proteases }\end{array}$ \\
\hline & Phylomer ${ }^{\mathbb{R}}$ peptides & Published in vitro studies & Inhibits $C p$ IMPDH \\
\hline & $\begin{array}{l}\text { Compounds from } \\
\text { NIH Clinical Collections }\end{array}$ & Published in vitro studies & $\begin{array}{l}\text { Two compounds from } \\
\text { this collection have } \\
\text { been tested }\end{array}$ \\
\hline & $\begin{array}{l}\text { Malaria box drug-like } \\
\text { compounds }\end{array}$ & One published in vitro study & \\
\hline & $\begin{array}{l}\text { Auronofin and other } \\
\text { "orphan drugs" }\end{array}$ & $\begin{array}{l}\text { Several published in vitro } \\
\text { studies }\end{array}$ & $\begin{array}{l}\text { Auranolin has been } \\
\text { successfully tested } \\
\text { against other } \\
\text { apicomplexans in vivo }\end{array}$ \\
\hline
\end{tabular}

response and neuropeptides such as substance $\mathrm{P}$ may be key contributors $[8,9]$.

The burden of disease caused by Cryptosporidium worldwide has been significantly underestimated. For example, only about $1 \%$ of the estimated 750,000 cases that occur annually in the US are reported [10, 11]. Historically, Cryptosporidium was thought of primarily as a cause of chronic diarrhea in AIDS and other immunocompromised patients. More recent data has shed light on the parasite's effect on children in resource-poor areas. Older studies, using acid fast staining to identify the organisms, found Cryptosporidium in $<5 \%$ of cases of childhood diarrhea. However, more recent studies using antigen and molecular assay have detected Cryptosporidium infection in 15-20\% of all childhood diarrhea $[1,12]$. In a multicenter study of childhood diarrhea in Sub-Saharan Africa and South Asia, Cryptosporidium was second to rotavirus as a cause of moderate-to-severe diarrhea in children 2 years of age and was a major cause of morbidity in the second year of life [13••]. Subsequent studies using molecular methods demonstrated that even that study had underdiagnosed cryptosporidiosis [14••].

There are also chronic sequelae of Cryptosporidium infection. Lima et al. showed that infection in children less than
1 year old was followed by recurrent diarrhea and growth stunting that continued for 2 years after the initial episode [15]. Follow-up studies demonstrated deficits in cognitive development and physical fitness when patients were examined 5 years later [16].

Cryptosporidiosis is more frequent and severe disease in children with malnutrition, including higher incidence of deaths $[2 \bullet \bullet, 17,18]$. Malnutrition predisposes patients to infection and creates a vicious infection-malnutrition cycle with long-term consequences including cognitive impairment and stunting [19]. Using an animal model that closely resembles the complex interaction between nutritional status and infection, Costa et al. showed $20 \%$ additional weight loss when malnourished mice were infected with $C$. parvum, higher fecal shedding and failure to prevent weight loss, or parasite stool shedding despite treatment with nitazoxanide [20]. In addition, children under the age of 1 year with Cryptosporidium infections fail to have catch-up growth that is typically observed with children infected at a later age. The diarrheal morbidity for this young age group is significantly increased as well [18, 21].

Symptomatic therapy is vital in cases of cryptosporidiosis. Replacement of fluids and electrolytes in cryptosporidiosis is 
critically important as in other causes of diarrhea. Antimotility drugs are also a key element of therapy. Most published patient studies utilize narcotic agents such as loperamide and diphenoxylate/atropine. Other reports suggest that tincture of opium (paregoric) may be a more effective agent in AIDS patients. Nutritional support is also imperative for successful treatment, which includes continued breastfeeding of infant patients.

Because cryptosporidiosis is typically self-limited in immunocompetent hosts, restoration of immune function is a key component of management. Immune reconstitution in response to effective combination antiretroviral therapy has been linked to parasite clearance, as well as reduced longterm morbidity and mortality associated with cryptosporidial infection of AIDS patients [2••, 22, 23]. Nevertheless, even with effective antiretroviral therapy, chronic diarrhea is associated with early mortality [24]. There are anecdotes of better responses when anti-motility and anti-parasitic drugs are used as part of the initial therapy [23]. However, there is no conclusive evidence that this is the case. Interestingly, some HIV protease inhibitors have activity against Cryptosporidium both in vitro and in vivo [25].

\section{Current Therapeutics}

Despite the fact that cryptosporidiosis has been recognized as an important cause of diarrheal disease for over three decades, anti-parasitic treatments have been limited. Initial screening of available compounds failed to identify effective treatments for cryptosporidiosis. A number of drugs previously reported to be effective have failed in clinical trials [22].

The only drug that has FDA approval for treatment of Cryptosporidium is nitazoxanide [2••]. Nitazoxanide was synthesized in the 1980s by combining a thiazole ring (similar structurally to metronidazole) with a benzamidine ring (similar to the tapeworm drug niclosamide). Nitazoxanide is a broad spectrum anti-parasitic with reports of use as a deworming agent as well as in controlled trials in giardiasis and cryptosporidiosis [22]. Three placebo-controlled trials of treatment of cryptosporidiosis with nitazoxanide in non-AIDS patients have been reported [26-28]. Studies reported up to $93 \%$ of treated patients experienced parasite clearance as opposed to $37 \%$ of placebo-treated patients [27]. The drug also has been shown to improve diarrhea and mortality rates among infected, malnourished children [26]. However, the response rate in malnourished children was only $56 \%$ [26]. Unfortunately, nitazoxanide has not been found to be effective in AIDS patients [29].

Nitazoxanide is only FDA approved for patients 1 year of age or older. A recent study among hospitalized children in Egypt aged 6 months to 10 years presenting with persistent diarrhea compared paromomycin and nitazoxanide with no antiparasitic treatment [30]. Overall, $86.6 \%$ of children treated with 100 or $200 \mathrm{mg}$ of nitazoxanide every $12 \mathrm{~h}$ for 3 days demonstrated complete clearance of oocysts and cessation of clinical symptoms. Among children treated with $25 \mathrm{mg} / \mathrm{kg} / \mathrm{day}$ of paromomycin for 2 weeks, $68.8 \%$ experienced stool clearance and were completely cured. Both treatments were better than no anti-parasitic treatment.

Though AIDS patients are the main immunocompromised population affected by Cryptosporidium, the parasite is also problematic in organ transplant recipients as well [31-33]. Bhadauria et al. conducted a retrospective review of living donor renal transplant recipients admitted for evaluation of diarrhea [33]. Cryptosporidium was found to be the most common cause of infectious diarrhea in these patients. Patients receiving combination immunosuppression including tacrolimus had higher rates of infection with Cryptosporidium compared to a combination of cyclosporine. Bhadauria and colleagues reported a better response to nitazoxanide/ flouroquinolone combination therapy than to nitazoxanide alone [33]. However, the authors did not test the patients for Escherichia coli enteropathogens. Thus, the benefit of the fluoroquinolone may have been on undiagnosed bacterial co-infection. Other groups have noted that transplant patients responded poorly to nitazoxanide alone. Still, there are anecdotes of better responses to combinations of nitazoxanide, azithromycin, and/or paromomycin [34-36].

Another population affected by chronic Cryptosporidium infections includes patients with primary immunodeficiencies such as hyper-IgM syndrome. This syndrome is a combined immune deficiency disorder caused by mutations in CD40 ligand. Infections in these patients are similar to that of AIDS patients, with extra-intestinal manifestations including biliary involvement. Fan et al. showed that treatments with CD40 agonist antibody effectively reduced the number of oocytes shed by these patients. However, relapse occurred when treatment was stopped [37].

Physicians initially approached the devastating effects of cryptosporidiosis in AIDS patients by testing a wide range of available drugs. One such drug, paromomycin, was reported to ameliorate cryptosporidiosis in AIDS patients [38]. In neither of the two controlled trials in patients with AIDS were the effects more than modest (few cures and mild decrease in diarrhea) $[38,39]$. In one controlled trial, there was a statistically significant decrease in oocyst shedding and diarrhea [40], while Hussien et al. demonstrated clearance of cryptosporidiosis among hospitalized children with paromomycin treatment compared to no treatment. However, the response was significantly less active than with nitazoxanide [30].

Other drugs reported to have some effect in case series include azithromycin, spiramycin, and bovine anticryptosporidium immunoglobulin [22]. However, all were ineffective in controlled trials in AIDS patients. Unfortunately, 
while all of these studies were presented at scientific meetings, none of these trials have been published [22]. Azithromycin seemed to be better than two anthelminthic drugs for cryptosporidiosis in children [41]. It has also been used in combination with nitazoxanide and/or paromomycin in compromised hosts with decreased stool frequency and parasite clearance in some patients [34, 42].

Rifamycins have been studied for anti-Cryptosporidium activity. Rifabutin was tested in vitro and showed a $25 \%$ decrease in C. parvum infection in vitro. When combined with nitazoxanide, infection decreased by $75 \%$ [43]. Holmberg et al. [44] reported an $85 \%$ decrease in the incidence of Cryptosporidium in the cohort of AIDS patients receiving rifabutin for $M$. avium complex prophylaxis, compared to the groups receiving clarithromycin or azithromycin. Similar results were seen when comparing AIDS patients taking rifabutin, clarithromycin, or a combination [45]. A small group of HIV patients were found to have resolution of diarrhea with rifaximin, a non-absorbed rifamycin [46, 47].

\section{Gaps}

While nitazoxanide was an important advance in the management of cryptosporidiosis in children, its limited efficacy in compromised or malnourished hosts has raised important questions regarding how to manage these patients. Clearly, we are in urgent need of better drugs for therapy of cryptosporidiosis. A pivotal step towards this goal is the identification of specific targets. At an experts' workshop on cryptosporidiosis, development of novel drugs for cryptosporidiosis was considered a critically important priority, with potentially huge public health payoffs. The inability to propagate the organisms in vitro or to genetically manipulate parasite gene expression

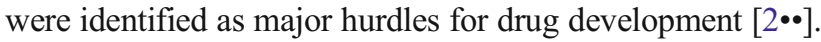

Progress in developing novel drugs against Cryptosporidium also has been hampered by limitations of current experimental models [48]. Because animal models are suboptimal for some human infections, human cell lines have been used as an alternative to study intestinal pathogens $[49,50]$. However, the utility of cell lines is limited by the fact they do not readily support parasite propagation. However, novel methods incorporating intestinal stem cells offer the prospect of significantly improving propagation [51].

Further hindrances in the research and treatment of Cryptosporidium are the gaps in understanding of the gastrointestinal and immune responses to the parasite. There is a specific lack of knowledge regarding the mechanisms of parasite clearance in immunocompetent hosts. Insight into this aspect of infection might enable advances in preventative research. A more in-depth knowledge of the gastrointestinal responses is also needed to facilitate optimization of current treatment methods as well as provide specific targets for preventatives.

\section{The Way Forward}

One of the typical difficulties faced when developing antiparasitic treatments is lack of drug targets that do not have human homologues. Several key enzymes have been identified as targets due to significant differences from human enzymes. Many apicomplexan parasites have unique calciumdependent protein kinases, including the Cryptosporidium CDPK1, an essential component of cell invasion [52]. The parasites lack amino acids blocking access to the active site by "bumped kinase inhibitors". Castellanos et al. have identified several compounds that bind to this enzyme, inhibiting enzyme function and ultimately killing the Cryptosporidium cell. A number of these inhibitors have exhibited anticryptosporidium activity both in vitro as well as in SCIDbeige immunocompromised mouse models [53].

Clan CA cysteine proteases have been found to be a potential target for the treatment of cryptosporidiosis. Clan CA cysteine proteases are thought to be vital for host cell invasion and have been found to be structurally different than analogous enzymes in humans [54]. This protease can be inhibited utilizing $N$-methyl-piperazine-Phe-homoPhe-vinylsulfone phenyl (K11777) inhibits growth of Cryptosporidium in vitro and has been shown to rescue immunocompromised mice from lethal infection [55].

The folate biosynthesis pathway, historically a target for cancer, bacterial, and malarial disease, has also been identified as a potential target for anti-cryptosporidials. Cryptosporidium contains a bi-functional thymidylate synthase/dihydrofolate reductase enzyme. Licensed antibacterial and anti-protozoan drugs do not inhibit the cryptosporidium enzyme, but research teams are evaluating the activity of compounds designed to specifically block this key enzyme in the folate synthesis pathway in vitro. Published data has reported striking anti-cryptosporidial activity of these compounds within cell culture [56].

Another potential drug target is oxidoreductase inosine 5'monophosphate dehydrogenase (IMPDH) which is essential in guanine synthesis in Cryptosporidium. Unlike human IMPDH, $C p$ IMPDH seems to have originated from bacteria via lateral gene transfer, thus the enzyme is structurally different than its human counterpart. Current research has found a series of inhibitors for this enzyme [57, 58]. Another study investigated the use of Phylomer ${ }^{\circledR}$ peptides to inhibit $\mathrm{Cp}$ IMPDH functions. These studies were conducted in vitro and identified two out of 12 peptides with anticryptosporidium functions [59].

The repurposing of already developed drugs or compounds is another promising area of anti-cryptosporidial research. A 
study done by Bessoff et al. utilized Human HMG-CoA Reductase and Isoprenoid Synthesis inhibitors from the NIH Clinical Collections drug library in a novel cell-based assay [60]. Results showed that there may be a surprising amount of overlap between previously FDA approved drugs and potential anti-cryptosporidal activity [60]. The use of Malaria Box drug-like compounds is also being investigated. These compounds have been created by the Medicines for Malaria Venture and are freely available to researchers [61]. A preliminary study identified several potential anti-cryptosporidiosis candidates; however, further in vitro and in vivo research has yet to be published [62]. Similarly, the anti-inflammatory drug auranofin demonstrates activity against Cryptosporidium in vitro; however, no in vivo or clinical results have been published [63].

The re-evaluation of current oral rehydration therapies is also being conducted to determine its role in the clearance of Cryptosporidium in immunocompetent hosts. Castro et al. demonstrated that supplementation of L-arginine to infected, undernourished mice aided in weight gain and reduced parasite burden [64]. Similar findings were reported by Costa et al. and Argenzio et al. utilizing the supplementation of an oligodeoxynucleotide with unmethylated $\mathrm{CpG}$ motif, alanylglutamine, and tumor necrosis factor alpha in in vitro and in vivo models $[65,66]$. These findings indicate that current recommended oral rehydration therapies may need to be updated; however, no clinical trials have been completed to asses these supplements in humans. Further research in this area could potentially provide a cost-effective treatment option for malnourished and immunocompromised patients in resource-limited areas.

\section{Conclusions}

Within the past few years, studies have increasingly focused on the importance of Cryptosporidium as a cause of childhood diarrhea and associated morbidity and mortality. However, there has been little clinical advancement in the treatment of cryptosporidiosis. The study conducted by Hussien et al. concluded that nitazoxanide treatment is superior to paromomycin in children with chronic diarrhea in endemic areas [30]. Successful development of novel drugs could also aid in decreasing childhood malnutrition. Progress has been slowed by limitation in methods to propagate the organisms in vitro and genetically manipulate the organisms. Nevertheless, research focused on anti-cryptosporidials continues to make substantial advancements. Several enzymes have been identified as potential drug targets, including calciumdependent protein kinases [53], Clan CA cysteine proteases [55], IMPDH [57], and the folate biosynthesis pathway [56]. Other studies are testing compounds repurposed, which were developed for other indications. However, none of these compounds have made it into clinical trials.

\section{Compliance with Ethics Guidelines}

Conflict of Interest Hayley Sparks, Gayatri Nair, and Alejandro Castellanos-Gonzalez declare that they have no conflict of interest.

A. Clinton White Jr receives royalties from UpToDate and Harrison's Internal Medicine for chapters on a different subject.

Human and Animal Rights and Informed Consent This article does not contain any studies with human subjects performed by any of the authors.

With regard to the authors' research cited in this paper, all institutional and national guidelines for the care and use of laboratory animals were followed.

\section{References}

Papers of particular interest, published recently, have been highlighted as:

•- Of major importance

1. White Jr AC. Cryptosporidiosis (Cryptosporidium species). In: Mandell D, editor. Bennett's principals and practices of infectious diseases. Philadelphia: Elsevier Saunders; 2015. p. 3173-83.e6.

2.• Checkley $\mathrm{W}$ et al. A review of the global burden, novel diagnostics, therapeutics, and vaccine targets for cryptosporidium. Lancet Infect Dis. 2015;15(1):85-94. This state of the art review came from a meeting of most of the leaders in the field to define the state-ofthe art, gaps, and future research directions regarding epidemiology, diagnosis, treatment, and vaccination for cryptosporidiosis.

3. Cama VA et al. Cryptosporidium species and subtypes and clinical manifestations in children, Peru. Emerg Infect Dis. 2008;14(10): 1567-74.

4. Ryan U, Fayer R, Xiao L. Cryptosporidium species in humans and animals: current understanding and research needs. Parasitology. 2014;141(13):1667-85.

5. Chalmers RM, Katzer F. Looking for Cryptosporidium: the application of advances in detection and diagnosis. Trends Parasitol. 2013;29(5):237-51.

6. Goodgame RW et al. Intestinal function and injury in acquired immunodeficiency syndrome-related cryptosporidiosis. Gastroenterology. 1995;108(4):1075-82.

7. Zhang $\mathrm{Y}$ et al. Lactulose-mannitol intestinal permeability test in children with diarrhea caused by rotavirus and cryptosporidium. Diarrhea Working Group, Peru. J Pediatr Gastroenterol Nutr. 2000;31(1):16-21.

8. Pantenburg B et al. Intestinal immune response to human Cryptosporidium sp. infection. Infect Immun. 2008;76(1):23-9.

9. Hernandez J et al. Substance P is responsible for physiological alterations such as increased chloride ion secretion and glucose malabsorption in cryptosporidiosis. Infect Immun. 2007;75(3): 1137-43.

10. Scallan E et al. Foodborne illness acquired in the United Statesmajor pathogens. Emerg Infect Dis. 2011;17(1):7-15.

11. Painter JE et al. Cryptosporidiosis surveillance-United States, 2011-2012. MMWR Surveill Summ. 2015;64 Suppl 3:1-14. 
12. Shirley DA, Moonah SN, Kotloff KL. Burden of disease from cryptosporidiosis. Curr Opin Infect Dis. 2012;25(5):555-63.

13.• Kotloff KL et al. Burden and aetiology of diarrhoeal disease in infants and young children in developing countries (the Global Enteric Multicenter Study, GEMS): a prospective, case-control study. Lancet. 2013;382(9888):209-22. This reports the results of a multicenter study of childhood diarrhea from 7 countries in SubSaharan Africa and South Asia. It highlighted the role of Cryptosporidium as second to rotavirus as a cause of moderate to severe diarrhea and death among children under 2 years of age.

14.• Liu J et al. Development and assessment of molecular diagnostic tests for 15 enteropathogens causing childhood diarrhoea: a multicentre study. Lancet Infect Dis. 2014;14(8):716-24. This multicenter study compared multiplex PCR, film array, and bead arrays assays for multiple pathogens. Overall, it demonstrated that each of the techniques was highly reproducible and demonstrated that Cryptosporidium was underdiagnosed even using specimens from the GEMS study.

15. Moore SR et al. Prolonged episodes of acute diarrhea reduce growth and increase risk of persistent diarrhea in children. Gastroenterology. 2010;139(4):1156-64.

16. Guerrant DI et al. Association of early childhood diarrhea and cryptosporidiosis with impaired physical fitness and cognitive function four-seven years later in a poor urban community in northeast Brazil. Am J Trop Med Hyg. 1999;61(5):707-13.

17. Mondal $\mathrm{D}$ et al. Contribution of enteric infection, altered intestinal barrier function, and maternal malnutrition to infant malnutrition in Bangladesh. Clin Infect Dis. 2012;54(2):185-92.

18. Molbak $\mathrm{K}$ et al. Cryptosporidium infection in infancy as a cause of malnutrition: a community study from Guinea-Bissau, West Africa. Am J Clin Nutr. 1997;65(1):149-52.

19. Guerrant RL et al. The impoverished gut - a triple burden of diarrhoea, stunting and chronic disease. Nat Rev Gastroenterol Hepatol. 2013;10(4):220-9.

20. Costa LB et al. Cryptosporidium-malnutrition interactions: mucosal disruption, cytokines, and TLR signaling in a weaned murine model. J Parasitol. 2011;97(6):1113-20.

21. Checkley W et al. Effects of Cryptosporidium parvum infection in Peruvian children: growth faltering and subsequent catch-up growth. Am J Epidemiol. 1998;148(5):497-506.

22. Cabada MM, White Jr AC. Treatment of cryptosporidiosis: do we know what we think we know? Curr Opin Infect Dis. 2010;23(5): 494-9.

23. Masur $\mathrm{H}$ et al. Prevention and treatment of opportunistic infections in HIV-infected adults and adolescents: updated guidelines from the Centers for Disease Control and Prevention, National Institutes of Health, and HIV Medicine Association of the Infectious Diseases Society of America. Clin Infect Dis. 2014;58(9):1308-11.

24. Dillingham RA et al. High early mortality in patients with chronic acquired immunodeficiency syndrome diarrhea initiating antiretroviral therapy in Haiti: a case-control study. Am J Trop Med Hyg. 2009;80(6):1060-4.

25. Mele $\mathrm{R}$ et al. Indinavir reduces Cryptosporidium parvum infection in both in vitro and in vivo models. Int J Parasitol. 2003;33(7):75764.

26. Amadi B et al. Effect of nitazoxanide on morbidity and mortality in Zambian children with cryptosporidiosis: a randomised controlled trial. Lancet. 2002;360(9343):1375-80.

27. Rossignol JF et al. Effect of nitazoxanide in diarrhea and enteritis caused by Cryptosporidium species. Clin Gastroenterol Hepatol. 2006:4(3):320-4.

28. Rossignol JF, Ayoub A, Ayers MS. Treatment of diarrhea caused by Cryptosporidium parvum: a prospective randomized, double-blind, placebo-controlled study of Nitazoxanide. J Infect Dis. 2001;184(1):103-6.
29. Amadi B et al. High dose prolonged treatment with nitazoxanide is not effective for cryptosporidiosis in HIV positive Zambian children: a randomised controlled trial. BMC Infect Dis. 2009;9:195.

30. Hussien SM et al. Comparative study between the effect of nitazoxanide and paromomycine in treatment of cryptosporidiosis in hospitalized children. J Egypt Soc Parasitol. 2013;43(2):463-70.

31. Raja K et al. Prevalence of cryptosporidiosis in renal transplant recipients presenting with acute diarrhea at a single center in Pakistan. J Nephropathol. 2014;3(4):127-31.

32. Danziger-Isakov L. Gastrointestinal infections after transplantation. Curr Opin Gastroenterol. 2014;30(1):40-6.

33. Bhadauria $\mathrm{D}$ et al. Cryptosporidium infection after renal transplantation in an endemic area. Transpl Infect Dis. 2015;17(1):48-55.

34. Legrand F et al. Diagnosis and treatment of digestive cryptosporidiosis in allogeneic haematopoietic stem cell transplant recipients: a prospective single centre study. Bone Marrow Transplant. 2011;46(6):858-62.

35. Bonatti $\mathrm{H}$ et al. Cryptosporidium enteritis in solid organ transplant recipients: multicenter retrospective evaluation of 10 cases reveals an association with elevated tacrolimus concentrations. Transpl Infect Dis. 2012;14(6):635-48.

36. Krause I et al. Cryptosporidiosis in children following solid organ transplantation. Pediatr Infect Dis J. 2012;31(11):1135-8.

37. Fan $\mathrm{X}$ et al. CD40 agonist antibody mediated improvement of chronic Cryptosporidium infection in patients with X-linked hyper IgM syndrome. Clin Immunol. 2012;143(2):152-61.

38. Hashmey R et al. Cryptosporidiosis in Houston, Texas. A report of 95 cases. Medicine (Baltimore). 1997;76(2):118-39.

39. Hewitt RG et al. Paromomycin: no more effective than placebo for treatment of cryptosporidiosis in patients with advanced human immunodeficiency virus infection. AIDS Clinical Trial Group. Clin Infect Dis. 2000;31(4):1084-92.

40. White Jr AC et al. Paromomycin for cryptosporidiosis in AIDS: a prospective, double-blind trial. J Infect Dis. 1994;170(2):419-24.

41. Allam AF, Shehab AY. Efficacy of azithromycin, praziquantel and mirazid in treatment of cryptosporidiosis in school children. J Egypt Soc Parasitol. 2002;32(3):969-78.

42. Smith $\mathrm{NH}$ et al. Combination drug therapy for cryptosporidiosis in AIDS. J Infect Dis. 1998;178(3):900-3.

43. Giacometti A et al. Activity of nitazoxanide alone and in combination with azithromycin and rifabutin against Cryptosporidium parvum in cell culture. J Antimicrob Chemother. 2000;45(4):4536.

44. Holmberg SD et al. Possible effectiveness of clarithromycin and rifabutin for cryptosporidiosis chemoprophylaxis in HIV disease. HIV Outpatient Study (HOPS) Investigators. JAMA. 1998;279(5): 384-6.

45. Fichtenbaum CJ et al. Rifabutin but not clarithromycin prevents cryptosporidiosis in persons with advanced HIV infection. AIDS. 2000;14(18):2889-93.

46. Amenta $\mathrm{M}$ et al. Intestinal protozoa in HIV-infected patients: effect of rifaximin in Cryptosporidium parvum and Blastocystis hominis infections. J Chemother. 1999;11(5):391-5.

47. Gathe Jr JC et al. Resolution of severe cryptosporidial diarrhea with rifaximin in patients with AIDS. J Acquir Immune Defic Syndr. 2008;48(3):363-4.

48. Kothavade RJ. Challenges in understanding the immunopathogenesis of Cryptosporidium infections in humans. Eur J Clin Microbiol Infect Dis. 2011;30(12):1461-72.

49. Feng $\mathrm{H}$ et al. Bile acids enhance invasiveness of Cryptosporidium spp. into cultured cells. Infect Immun. 2006;74(6):3342-6.

50. Yang YL, Buck GA, Widmer G. Cell sorting-assisted microarray profiling of host cell response to Cryptosporidium parvum infection. Infect Immun. 2010;78(3):1040-8. 
51. Castellanos-Gonzalez A et al. Human primary intestinal epithelial cells as an improved in vitro model for Cryptosporidium parvum infection. Infect Immun. 2013;81(6):1996-2001.

52. Murphy RC et al. Discovery of potent and selective inhibitors of calcium-dependent protein kinase 1 (CDPK1) from C. parvum and T. gondii. ACS Med Chem Lett. 2010;1(7): 331-5.

53. Castellanos-Gonzalez A et al. A novel calcium-dependent protein kinase inhibitor as a lead compound for treating cryptosporidiosis. J Infect Dis. 2013;208(8):1342-8.

54. Na BK et al. Cryptopain-1, a cysteine protease of Cryptosporidium parvum, does not require the pro-domain for folding. Parasitology. 2009;136(2):149-57.

55. Ndao $\mathrm{M}$ et al. A cysteine protease inhibitor rescues mice from a lethal Cryptosporidium parvum infection. Antimicrob Agents Chemother. 2013;57(12):6063-73.

56. Kumar VP et al. Structural studies provide clues for analog design of specific inhibitors of Cryptosporidium hominis thymidylate synthase-dihydrofolate reductase. Bioorg Med Chem Lett. 2014;24(17):4158-61.

57. Sun $Z$ et al. Synthesis, in vitro evaluation and cocrystal structure of 4-oxo-[1]benzopyrano[4,3-c]pyrazole Cryptosporidium parvum inosine 5'-monophosphate dehydrogenase (CpIMPDH) inhibitors. J Med Chem. 2014;57(24):10544-50.

58. Gorla SK et al. Validation of IMP dehydrogenase inhibitors in a mouse model of cryptosporidiosis. Antimicrob Agents Chemother. 2014;58(3):1603-14.
59. Jefferies $\mathrm{R}$ et al. Target validation of the inosine monophosphate dehydrogenase (IMPDH) gene in Cryptosporidium using Phylomer((R)) peptides. Exp Parasitol. 2015;148:40-8.

60. Bessoff $\mathrm{K}$ et al. Drug repurposing screen reveals FDA-approved inhibitors of human HMG-CoA reductase and isoprenoid synthesis that block Cryptosporidium parvum growth. Antimicrob Agents Chemother. 2013;57(4):1804-14.

61. Spangenberg T et al. The open access malaria box: a drug discovery catalyst for neglected diseases. PLoS One. 2013;8(6), e62906.

62. Bessoff $\mathrm{K}$ et al. Identification of Cryptosporidium parvum active chemical series by repurposing the open access malaria box. Antimicrob Agents Chemother. 2014;58(5):2731-9.

63. Debnath A, Ndao M, Reed SL. Reprofiled drug targets ancient protozoans: drug discovery for parasitic diarrheal diseases. Gut Microbes. 2013;4(1):66-71.

64. Castro IC et al. Arginine decreases Cryptosporidium parvum infection in undernourished suckling mice involving nitric oxide synthase and arginase. Nutrition. 2012;28(6):678-85.

65. Costa LB et al. Novel in vitro and in vivo models and potential new therapeutics to break the vicious cycle of Cryptosporidium infection and malnutrition. J Infect Dis. 2012;205(9):1464-71.

66. Kandil HM, Berschneider HM, Argenzio RA. Tumour necrosis factor alpha changes porcine intestinal ion transport through a paracrine mechanism involving prostaglandins. Gut. 1994;35(7):934 40. 